ITC $1 / 49$

Information Technology and Control

Vol. 49 / No. $1 / 2020$

pp. $80-88$

DOI /10.5755/j01.itc.49.1.22855
Mismatch Removal Based on Gaussian Mixture Model for

Aircraft Surface Texture Mapping

Accepted after revision 2019/11/19

HOW TO CITE: Niu, G., Wang, L., \& Tan, Z. (2020). Mismatch Removal Based on Gaussian Mixture Model for Aircraft Surface Texture Mapping. Information Technology and Control, 49(1), 80-88. https://doi.org//10.5755/j01.itc.49.1.22855

\title{
Mismatch Removal Based on Gaussian Mixture Model for Aircraft Surface Texture Mapping
}

\section{Guochen Niu}

Robotics Institute, College of Electronic Information and Automation, Civil Aviation University of China, Tianjin 300300, China; phone: +86-13752255179; e-mail: niu_guochen@139.com

\section{Licheng Wang}

Robotics Institute, College of Electronic Information and Automation, Civil Aviation University of China, Tianjin 300300, China; phone: +86-13623658871; e-mail: licheng_wang@139.com

\section{Zheng Tan}

Robotics Institute, College of Electronic Information and Automation, Civil Aviation University of China, Tianjin 300300, China; phone: +86-15525527365; e-mail:2111055015@qq.com

Corresponding author: licheng_wang@139.com

Aiming at the lower efficiency and higher time cost for feature matching in aircraft surface texture mapping process, a novel mismatch removal method based on Gaussian mixture model is proposed to increase correct corresponding feature matching point pairs. The detection and initial point sets for corresponding pairs are carried out, and a vector field is interpolated between the two matching of (Oriented FAST and Rotated BRIEF) ORB feature points. The Gaussian mixture model (GMM) is introduced and the prior information is utilized to force the smoothness of the field, which is based on the Tikhonov regularization in vector-valued reproducing kernel Hilbert space (RKHS). In order to obtain the optimal estimation, the MAP solution of a Bayesian model with latent variables, which could be performed by Expectation Maximization (EM) algorithm, is utilized to determine the correct correspondence. The experimental results show that the algorithm could remove mismatches effectively and the classification for feature points is excellent. Moreover, 
the calculation time is greatly reduced, which enhanced the real-time performance of aircraft surface texture mapping process.

KEYWORDS: Mismatch removal; Gaussian Mixture Model; Tikhonov regularization; EM algorithm; Vector field.

\section{Introduction}

Aircraft surface texture mapping needs to map the images captured by camera to the three-dimensional pattern to obtain the reconstruction model with detail information, which could assist the crew to perform aircraft peripheral interested area detection. It becomes critical to match the corresponding feature points correctly, which could affect the crew to make an accurate judgment on the condition of the aircraft. In order to obtain the high-efficiency matching results, it is necessary to conduct a further study on mismatch algorithm.

Establishing reliable correspondence between images is an important computer vision aspect with numerous applications, including 3D surface reconstruction, image registration, robot localization and mapping, object recognition and motion estimation [12]. The above task still remains a challenge due to image noise, camera angle and illumination changes. It is necessary to study the registration accuracy of feature points. Several image matching techniques have been proposed so far. A popular strategy for solving the matching problem is to use a two-stage process [18]. The first stage is feature extraction and initial matching. The Harris corner detector, which relies on the extraction and tracking of feature points or corners, is a traditional method and has been widely used in simple images [10]. It is not suitable for images with detailed information. Scale-Invariant Feature Transform (SIFT) algorithm has been widely used as the mainstream algorithm for image matching because of its strong robustness to illumination and scale rotation $[9,14]$. However, the cost is an increase in computation time [8, 22]. With the increasing high demand for the matching speed, ORB algorithm was proposed, which is at two orders of magnitude faster than SIFT and one order of magnitude faster than SURF [23]. When feature extraction is accomplished, the correspondences are usually computed by using similarity constraint which would include a large number of false matches. The second stage is designed to remove the mismatches and estimate the geometric parame- ters. Various robust estimators have been proposed to distinguish correct correspondences from mismatches. Random Sample Consensus (RANSAC) algorithm is a typical approach for this strategy [3]. It tries to use four pairs of different matching points to get a minimum subset to estimate parametric model by re-sampling, and then determines the correct match point pair sets. The method is successful in many situations $[28,30,31]$. However, it has a limitation on the aircraft curved surface images. At present, vector field interpolation is used in computer vision and machine learning [24, 27]. A classical application of vector field interpolation is feature map in RKHS [5]. A number of methods about this area has developed based on regularization theory $[1,2]$. A multi-task vector field learning was proposed [16] and a new framework of regularization in the RKHS was developed about vector-valued function between input and output space, which could determine the relationship by choosing a suitable kernel $[19,20]$. However, these methods ignore the robustness issue. Taking into account these shortcomings, some scholars $[7,26]$ have introduced the technique of robust vector field interpolation into Gaussian process, which obtains the necessary and sufficient conditions for the vector to converge to the optimal estimator, but they do not consider the particularity of the images themselves, lacking specific applications in the images.

In summary, image registration has a great improvement in matching efficiency and computing time. In this paper, an effective method is introduced to perform image registration and remove the mismatch for aircraft surface texture mapping process. After feature detection and initial matches, a vector field is interpolated between the two points sets. Moreover, a Gaussian mixture model is used to introduce latent variables to ensure the smoothness of vector field so that the problem is transformed into maximum a posteriori estimation problem, which could be solved by EM algorithm and regularization method. The algorithm framework is described in Figure 1. 


\section{Figure 1}

The framework of the proposed algorithm

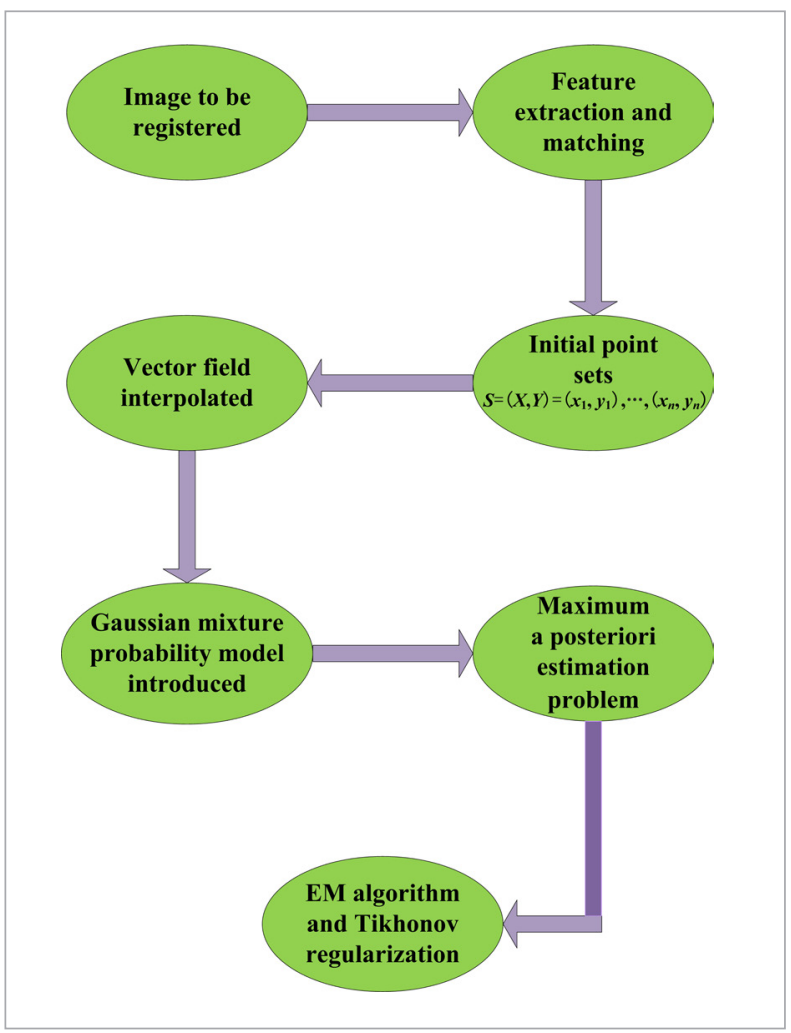

\section{Feature Extraction and Matching}

ORB feature descriptor has been widely used in feature matching because of its high-speed calculation and lower time cost. The method is built on the wellknown Oriented FAST feature point detector and recently-developed BRIEF descriptor, which shows good performance of rotation invariant and is resistant to noise. FAST feature point is convenient in extracting the characteristics, however, it does not have the direction information. The ORB algorithm adds the direction information to the FAST detector by obtaining the intensity centroid of the adjacent area about the feature points to confirm direction parameters. The neighborhood moment in an image block is defined as Equation (1):

$$
m_{\alpha \beta}=\sum_{x, y} x^{\alpha} y^{\beta} I(x, y),
$$

where $x, y$ are both relative FAST feature point loca- tion, and the circular radius of neighborhood is $r . x$, $y \in[-r, r], \alpha, \beta \in(0,1)$.

The centroid, recorded as $Q$, could be found as Equation (2):

$$
Q=\left(\frac{\sum x I(x, y)}{\sum I(x, y)}, \frac{\sum y I(x, y)}{\sum I(x, y)}\right)=\left(\frac{m_{10}}{m_{00}}, \frac{m_{01}}{m_{00}}\right) .
$$

Assuming that the center of the image block is $O$, a direction vector is constructed with the direction from center $O$ to the centroid $Q$. The deflection angle, denoted as $\Delta$, could represent the rotation angle, which is defined as Equation (3):

$$
\Delta=\arctan \left(\frac{\sum y I(x, y)}{\sum x I(x, y)}\right)=\left(\frac{m_{01}}{m_{10}}\right) .
$$

Based on the location and direction of the feature points, the BRIEF descriptor, a binary feature string description, could be extracted from the main direction. For the image block with smooth processing, the binary test $\xi$ is defined in the following form:

$$
\xi(P ; X, Y)=\left\{\begin{array}{ll}
1 & P(X)<P(Y) \\
0 & P(X) \geq P(Y)
\end{array},\right.
$$

where $P(\cdot)$ represents the intensity of block at the point (.). Then the binary feature string, of which the number is $n$, could be defined as a vector as follows:

$$
f_{n}(P)=\sum_{1 \leq i \leq n} 2^{i-1} \xi(P ; X, Y) .
$$

In the feature extraction, the circular area is used to determine whether the point is required, making the ORB algorithm obtains rotation invariance performance. Therefore, the ORB algorithm is more efficient in point detection because of the fast speed of calculating the intensity centroid.

After feature points were detected, Brute-Force Matcher was performed in feature matching. The main idea is to measure the descriptor distance, which could express the similarity between the two feature points. In other words, the distances between each feature point in one image and all feature points in another image are detected and arranged in the ascending order. Hamming distance is widely used in binary feature string. The idea is utilized to find the nearest and adjacent distance of the feature points, and the ratio of the two distance is compared with a threshold to determine whether the feature point pair is matched. 


\section{Mismatch Removal by Gaussian Mixture Model}

When initial match is accomplished, mismatches maybe exist, which may affect the accuracy of the aircraft surface texture mapping. It is necessary to select the correct corresponding pairs. Gaussian mixture model is an effective means, and is widely used in various fields $[13,29]$. In this paper, Gaussian mixture model was introduced to eliminate the mismatches and distinguish more true feature correspondences between images.

Let us suppose that the initial set of feature matching points extracted from two adjacent images is $S=$ $(X, Y)=\left(x_{1}, y_{1}\right), \ldots,\left(x_{N}, y_{N}\right)$, with $x_{n}$ and $y_{n}$ being the spatial positions of a correspondence in the two images. Due to the existence of mismatches, it is essential to obtain a robust estimation to remove the mismatches. In order to improve the performance of the algorithm, the images are both pre-processed to have zero mean and unit variance. To this end, a vector field $f$ is interpolated between the spatial point sets, which involve a consensus of correspond points. The key points in the sets are assumed to belong to a reproducing kernel Hilbert space. That is $f \in$ RKHS. The Gaussian mixture model, without loss of generality, is established and an assumption is built that the noise of corresponding points is Gaussian with zero mean and uniform standard deviation $\sigma$, while the distribution of mismatches is assumed to be uniform $\frac{1}{a}$ with $a$ being a constant. $\gamma$ is the percentage of corresponding points, and the mixture model of distribution is defined as follows:

$$
\begin{aligned}
p(X, Y \mid \theta) & =\prod_{n=1}^{N} p\left(x_{n}, y_{n} \mid \theta\right) \\
& =\prod_{n=1}^{N}\left[\frac{\gamma}{2 \pi \sigma^{2}} e^{-\frac{\left\|y_{n}-f\left(x_{n}\right)\right\|^{2}}{2 \sigma^{2}}}+\frac{1-\gamma}{a}\right],
\end{aligned}
$$

where $\theta=\left\{f, \sigma^{2}, \gamma\right\}$ is the set of unknown parameters, $X=\left(x_{1}, \ldots, x_{N}\right)^{T}, Y=\left(y_{1}, \ldots, y_{N}\right)^{T}$.

A hypothesis is formulated that $f$ is a random field with a known prior probability distribution, and the prior information of $f$ could be described as $p(f) \propto e^{-\frac{\lambda}{2}\|f\|^{2}}$, where $\lambda$ is a positive real number and $\|\cdot\|^{2}$ represents the square norm.
According to Bayes rules, the posterior distribution could be described as

$$
L(\theta)=p(\theta \mid X, Y) \propto p(X, Y \mid \theta) p(f) .
$$

In order to get the optimal solution of $\theta$, a Maximum a Posteriori (MAP) solution is introduced, which could be given by

$$
\hat{\theta}=\underset{\theta}{\arg \max } p(X, Y \mid \theta) p(f) .
$$

Thus, the vector field $f$ could be obtained from the optimal solution $\hat{\theta}$. Aiming at Equation (7), the log-likelihood function could be defined as

$l(\theta)=\ln L(\theta)=\ln p(\theta \mid X, Y)=\ln p(X, Y \mid \theta)+\ln p(f) .(9)$

Here, a latent variable $\mathrm{z}_{n} \in(0,1)$, associated with $n$-th sample, is introduced, where $z_{n}=1$ represents the Gaussian distribution and $\mathrm{z}_{n}=0$ represents the uniform distribution.

Then Equation (9) could be transformed into Equation (10) by omitting terms that are independent $\theta$ :

$$
\begin{aligned}
l(\theta) & =\ln \gamma \sum_{n=1}^{N} P\left(z_{n}=1 \mid x_{n}, y_{n}, \theta\right)-\ln 2 \pi \sigma^{2} \sum_{n=1}^{N} P\left(z_{n}=1 \mid x_{n}, y_{n}, \theta\right) \\
& -\frac{\left\|y_{n}-f\left(x_{n}\right)\right\|^{2}}{2 \sigma^{2}} \sum_{n=1}^{N} P\left(z_{n}=1 \mid x_{n}, y_{n}, \theta\right) \\
& +\ln (1-\gamma) \sum_{n=1}^{N} P\left(z_{n}=0 \mid x_{n}, y_{n}, \theta\right)-\frac{\lambda}{2}\|f\|^{2}
\end{aligned} .
$$

There are several ways to solve the parameters of Gaussian mixture model $[4,17,25]$. The EM algorithm with a natural framework is also widely used; it includes two steps: the expectation step (E-step) and the maximization step (M-step).

In the E-step, the current parameter $\theta$ is used to estimate the posterior distribution of the latent variables. $P=\operatorname{diag}\left(p_{1}, \ldots, p_{N}\right)$ is a diagonal matrix, and $p_{n}=P\left(\mathrm{z}_{n}=1 \mid x_{n}\right.$, $y_{n}, \theta$ ), which could be computed by Bayes rules:

$$
p_{n}=\frac{\gamma e^{-\frac{\left\|y_{n}-f\left(x_{n}\right)\right\|^{2}}{2 \sigma^{2}}}}{\gamma e^{-\frac{\left\|y_{n}-f\left(x_{n}\right)\right\|^{2}}{2 \sigma^{2}}}+\frac{2 \pi \sigma^{2}(1-\gamma)}{a}} .
$$


According to Equation (11), the posterior probability $p_{n}$ indicates to what degree the sample $n$ agrees with the current estimation of $f$.

In the M-step, the aim is to update the estimated parameters. Deriving Equation (10) with respect to $\sigma^{2}$ and $\gamma$, and setting them to zero, their matrix form could be expressed as Equation (12):

$$
\left\{\begin{array}{l}
\sigma^{2}=\frac{\operatorname{tr}\left[(Y-V)^{T} P(Y-V)\right]}{2 \cdot \operatorname{tr}(P)} \\
\gamma=\frac{\operatorname{tr}(P)}{N}
\end{array},\right.
$$

where $V=\left(f\left(x_{1}\right), \ldots, f\left(x_{n}\right)\right)^{T}$ and $\operatorname{tr}(\cdot)$ is the trace.

According to Equation (10), the variables, which are not related to parameter $f$, are taken to be ignored, and the remaining components are multiplied by -1 , so that the deformation could be written as follows:

$$
\iota(f)=\frac{\left\|y_{n}-f\left(x_{n}\right)\right\|^{2}}{2 \sigma^{2}} \sum_{n=1}^{N} p_{n}+\frac{\lambda}{2}\|f\|^{2} .
$$

In contrast to Equation (10), the maximization of $l(\theta)$ respected to $f$ could be equivalent to minimizing $\imath(f)$. Equation (13) is a typical Tikhonov regularization form $[6,11,15,21]$, which is the so-called the regularized risk function. The first term is the empirical risk, which measures the price when $f\left(x_{n}\right)$ replaces $y_{n}$, and the second term is squared norm in a RKHS, which enforces smoothness to the vector field $f$.

By choosing different kernels, the norm in the corresponding RKHS could determine different smoothness. Here, a Gaussian kernel as $k\left(x_{i}, x_{j}\right)=e^{-\beta\left\|x_{i}-x_{j}\right\|^{2}}$ is chosen. Then the optimal $f$ could be described as Equation (14):

$$
f(x)=\sum_{n=1}^{N} \Gamma\left(x, x_{n}\right) c_{n},
$$

where $\Gamma$ is a positive definite block matrices with $N \times N$, and the $(i, j)$-th block is $\Gamma\left(x_{i}, x_{i}\right)$, which could be defined as $\Gamma\left(x_{i}, x_{j}\right)=k\left(x_{i}, x_{j}\right) \cdot \mathrm{I}=e^{-\beta\left\|x_{i}-x_{j}\right\|^{2}} \cdot \mathrm{I}$. The coefficient $c_{n}$ is determined by a linear system

$$
\left(K+\lambda \sigma^{2} P^{-1}\right) C=Y,
$$

where $K$ is the Gram matrix with $K_{i j}=k\left(x_{i}, x_{j}\right)$, and $C=\left(c_{1}, \ldots, c_{N}\right)^{T}$ is the coefficient set.
When the EM algorithm converges, the optimal vector field $f$ would be obtained. Simultaneously, a threshold $\theta$ is set up, once the relationship of $p_{n}>\theta$ is confirmed, the correct corresponding point pairs are selected.

\section{Experiment and Result Analysis}

The images for the aircraft curved surface model are sampled. The ORB algorithm is used to extract feature points and the result is shown in Figure 2.

Figure 2

Feature points extracted by ORB algorithm

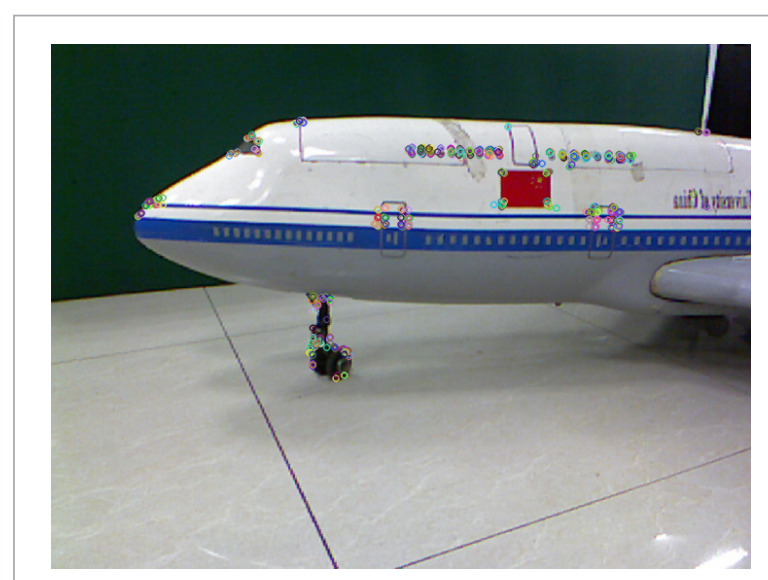

(a) Feature points for image I

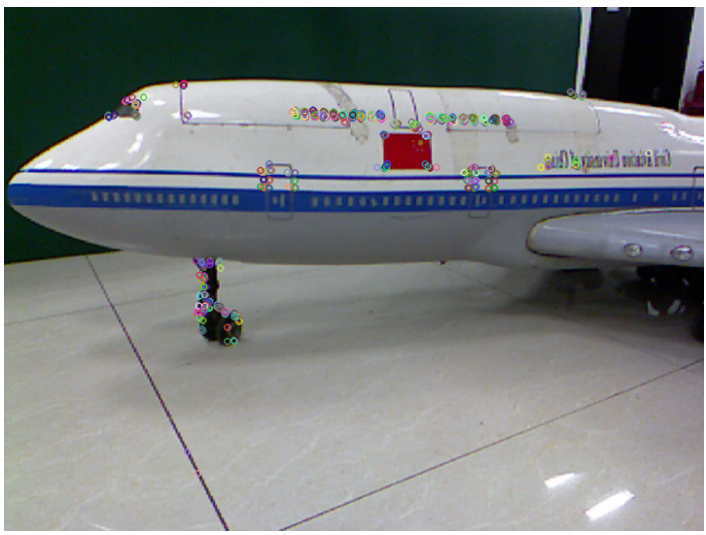

(b) Feature points for image II

As shown in Figure 3, some mismatches still exist after the initial match and the vector filed between the points are messy. In Figure 4, when Gaussian mixture model and Tikhonov regularization were introduced, the vec- 


\section{Figure 3}

The initial match and the messy vector field (a) The initial match point pairs, (b) The messy vector field

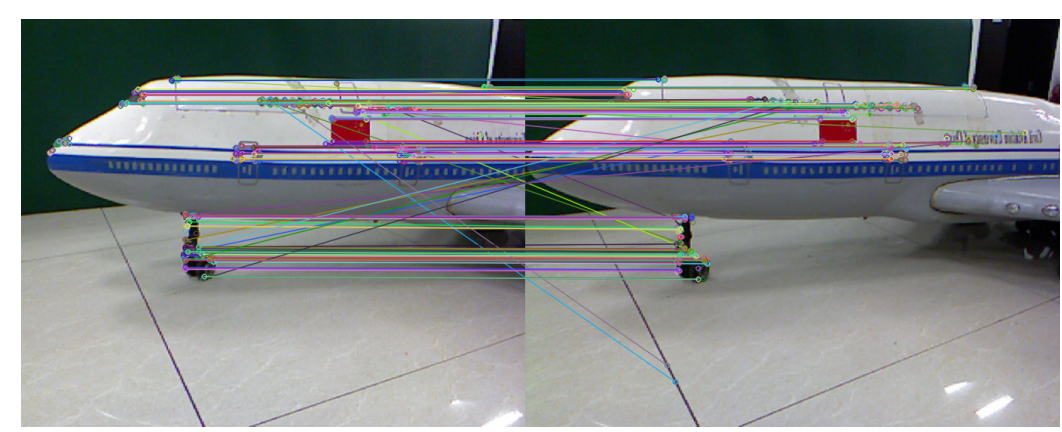

(a) The initial match point pairs

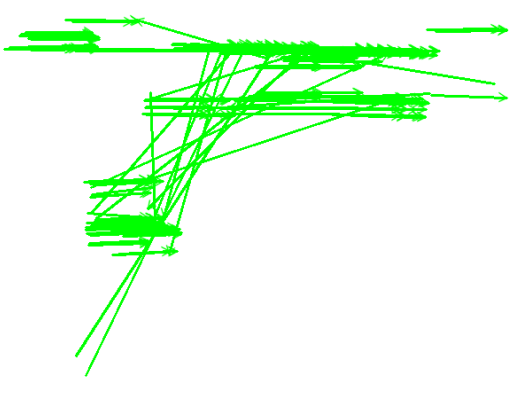

(b) The messy vector field

Figure 4

The correct corresponding matches and the smooth vector field (a) The correct corresponding matches, (b) The smooth vector field
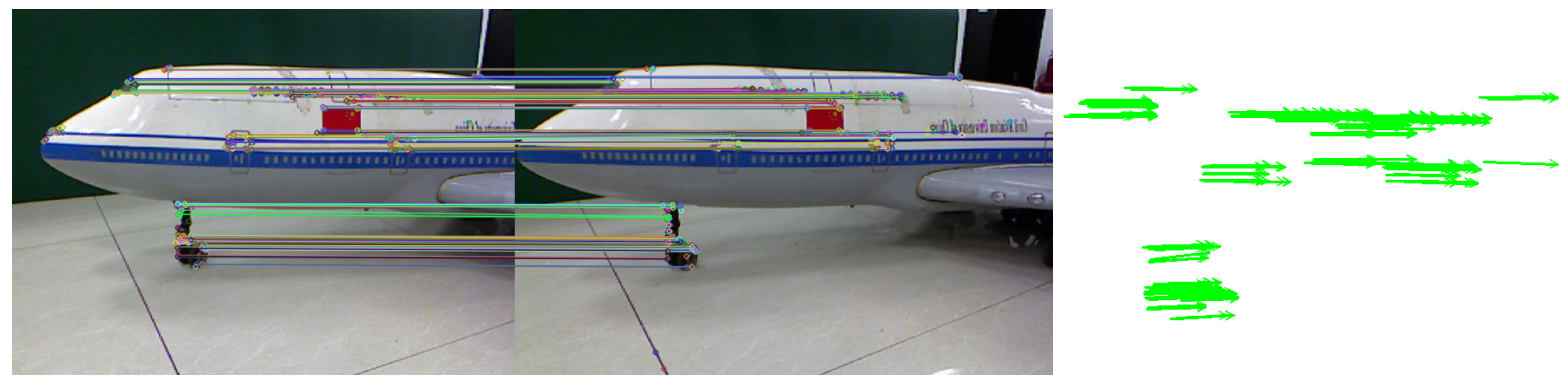

tor field turned to be smooth and consistent, and the correct corresponding matched pairs could be identified.

In order to verify the effectiveness of the proposed algorithm, the test is performed on a laptop with 1.6 GHz Intel Core CPU, 4GB memory and Microsoft Visual Studio code. When the threshold $\theta$ is defined as 0.7, the confusion matrix is performed as Table 1. Compared with the RANSAC algorithm, the time-consuming results are shown in Table 2.

\section{Table 1}

The confusion matrix for the GMM algorithm

\begin{tabular}{l|c|c|c}
\hline \multirow{2}{*}{} & \multicolumn{2}{c}{ Predicted class } \\
\cline { 3 - 4 } & & Positive & Negative \\
\hline \multirow{3}{*}{ Actual class } & Positive & 276 & 30 \\
\cline { 2 - 4 } & Negative & 52 & 162 \\
\hline
\end{tabular}

\section{Table 2}

The time-consuming results between GMM and RANSAC

\begin{tabular}{c|c|c}
\hline Time/(ms) & ORB+GMM & $\begin{array}{c}\text { SIFT+ } \\
\text { RANSAC }\end{array}$ \\
\hline Feature detection time & 1620.32 & 2138.13 \\
\hline Initial match time & 63.36 & 103.37 \\
\hline Mismatch removal time & 1981 & 2863 \\
\hline
\end{tabular}

It can be seen in Table 1 that the most of data are distributed on the main diagonal in the confusion matrix, showing that the classifier has a good classification effect. The calculation time cost for the algorithm in Table 2 is less, indicting a better real-time performance. For the purpose of further illustrating the feasibility of the algorithm, different confusion matrices are obtained by changing the model parameters, and such 
indexes as Precision, Recall and F-Measure are calculated. The test is carried out 35 times, and the indicators formed a series of scatter plots. The corresponding curves are drawn by using polynomial interpolation. The Precision-Recall curve and the F-Measure curve for the two algorithms are respectively shown in Figures 5 and 6. From Figure 5 , it can be seen that the data are concentrated in the upper right corner about the GMM method, and the index in the proposed algorithm is higher than that in RANSAC, indicating that the algorithm could achieve higher recall rate while having

\section{Figure 5}

The Precision-Recall curve of GMM and RANSAC

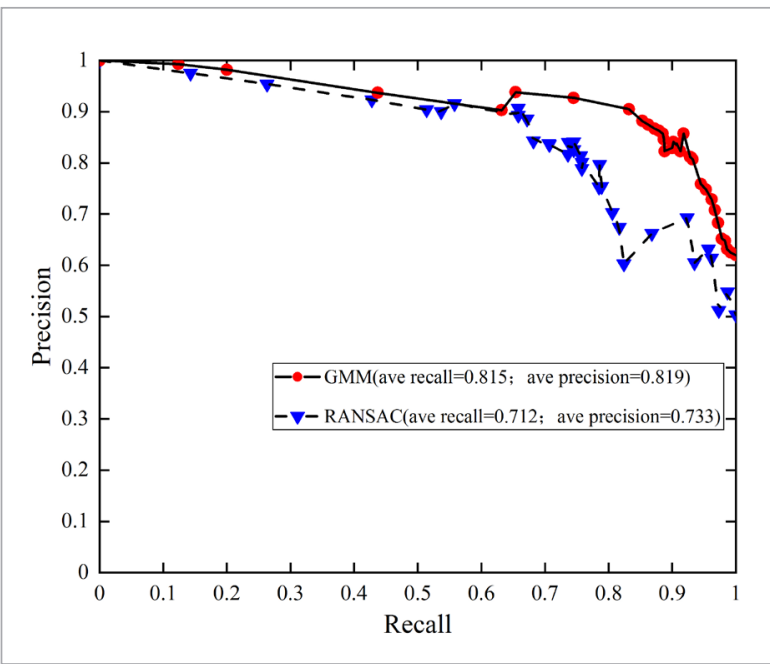

\section{Figure 6}

The F-Measure curve of GMM and RANSAC

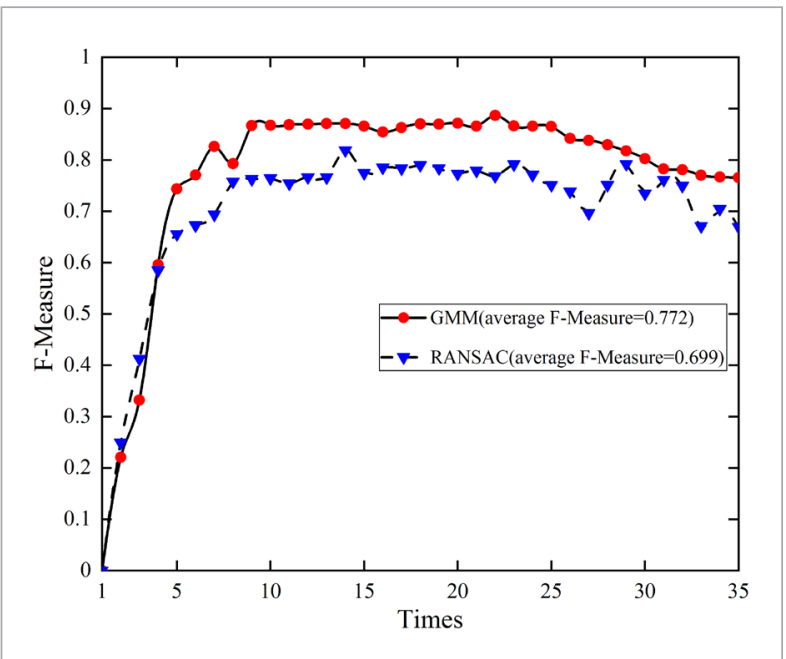

higher accuracy. According to Figure 6, the F-Measure curve for the GMM algorithm is above, demonstrating that the algorithm is more efficient. Figure 7 is the Receiver Operating Characteristic curve (ROC) for the two algorithms, and the index in the proposed algorithm is closer to the upper left corner, and its Area Under ROC curve (AUC) has a larger value, further indicating that the classification effect is better.

\section{Figure 7}

The ROC curve of GMM and RANSAC

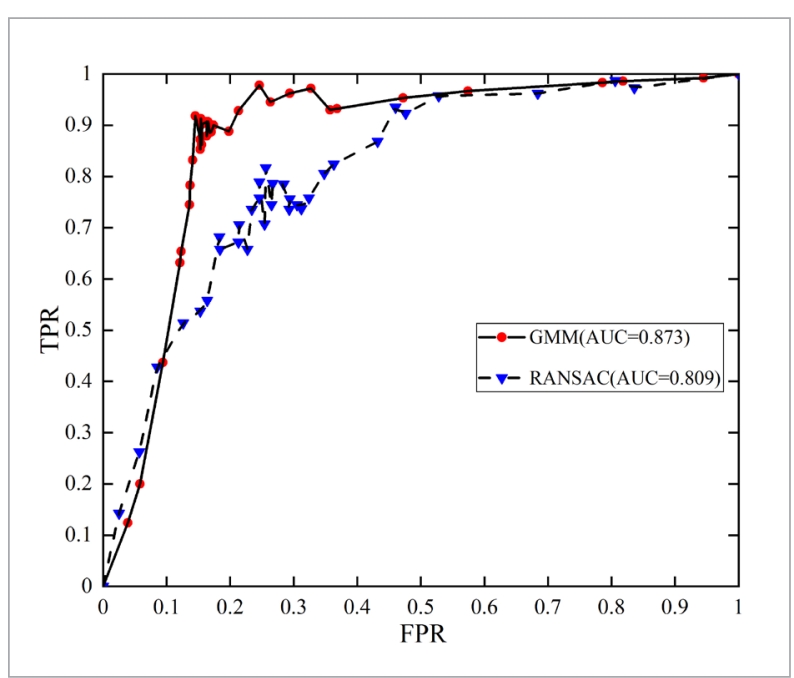

\section{Conclusion}

In this paper, a Gaussian mixture model with a vector field is studied on the characteristics of aircraft surface images. The real-time of the algorithm has been significantly ameliorated and the ability for the mismatch removal has been greatly improved. Experiments show that the proposed algorithm has good classification effect and the indicators are better. The applicability of the algorithm is well suitable to the image of aircraft curved surface model.

\section{Acknowledgement}

This work was funded by Tianjin Research Program of Application Foundation and Advanced Technology (\#14JCQNJC04400). The authors would like to thank their colleagues at the Robotics Institute of Civil Aviation University of China for their significant contributions. 


\section{References}

1. Abhishake, Sivananthan, S. Multi-Penalty Regularization in Learning Theory. Journal of Complexity, 2016, 36, 141-165. https://doi.org/10.1016/j.jco.2016.05.003

2. Baldassarre, L., Rosasco, L., Barla, A. Multi-Output Learning via Spectral Filtering. Machine Learning, 2012, 87(3), 259-301. https://doi.org/10.1007//s10994-012$5282-\mathrm{y}$

3. Bhattacharya, P., Gavrilova, M. Improving RANSAC Feature Matching with Local Topological Information. Proceedings of the 2012 9th International Symposium on Voronoi Diagrams in Science and Engineering, (ISVD 2012), New Brunswick, NJ, USA, June 27-29, 2012, 17-23. https://doi.org/10.1109/ISVD.2012.8

4. Bochinski, E., Jongebloed, R., Tok, M. Regularized Gradient Descent Training of Steered Mixture of Experts for Sparse Image Representation. 25th IEEE International Conference on Image Processing, (ICIP 2018), Athens, Greece, October 7-10, 2018, 3873-3877.https:// doi.org/10.1109/ICIP.2018.8451823

5. Celine, B., Szafranski, M., D’Alche-Buc, F. Input Output Kernel Regression: Supervised and Semi-Supervised Structured Output Prediction with Operator-Valued Kernels. Journal of Machine Learning Research, 2016, $17,1-48$.

6. Chung, J., Español, M. I. Learning Regularization Parameters for General-Form Tikhonov. Inverse Problems, 2017, 33(7). https://doi.org/10.1088/1361$6420 / 33 / 7 / 074004$

7. Du, J., Ma, S., Wu, Y. Convergence Analysis of Distributed Inference with Vector-Valued Gaussian Belief Propagation. Journal of Machine Learning Research, 2018, 18, 1-38.

8. Hossein-nejad, Z., Nasri, M. An Adaptive Image Registration Based on SIFT Features and Adaptive RANSAC Transform. Computers and Electrical Engineering, 2017, 62, 524-537. https://doi.org/10.1016/j.compeleceng.2016.11.034

9. Jiang, D., Yi, J. Comparison and Study of Classic Feature Point Detection Algorithm. 2012 International Conference on Computer Science and Service System, Nanjing, China, August 11-13, 2012, 2307-2309. https:// doi.org/10.1109/CSSS.2012.572

10. Jin, D., Zhu, S., Cheng, Y. Salient Object Detecction via Detection Harris Corner. 2017 29th Chinese Control and Decision Conference (CCDC 2017), Chongqing, China, May 28-30, 2017, 1108-1112. https://doi. org/10.1109/CCDC.2017.7978684
11. Jorgensen, P. E. T., Pearse, E. P. J. Continuum Versus Discrete Networks, Graph Laplacians, and Reproducing Kernel Hilbert Spaces. Journal of Mathematical Analysis and Applications, 2018, 469(2), 765-807. https://doi. org/10.1016/j.jmaa.2018.09.035

12. Kordelas, G., Daras, P. Robust SIFT-Based Feature Matching Using Kendall's Rank Correlation Measure. 16th IEEE International Conference on Image Processing, (ICIP 2009), Cairo, Egypt, November 07-10, 2009, 325328. https://doi.org/10.1109/ICIP.2009.5413514

13. Lee, K. H., Xue, L. Nonparametric Finite Mixture of Gaussian Graphical Model. Technometrics, 2017, 11. https://doi.org/10.1080/00401706.2017.1408497

14. Leng, C., Zhang, H., Li, B. Local Feature Descriptor for Image Matching: A Survey. IEEE Access, 2019, 7, 64246434. https://doi.org/10.1109/ACCESS.2018.2888856

15. Lewis, B., Reichel, L. Arnoldi-Tikhonov Regularization Methods. Journal of Computational and Applied Mathematics, 2009, 226(1), 92-102. https://doi.org/10.1016/j.cam.2008.05.003

16. Lin, B., Yang, S., Zhang, C. Multi-task Vector Field Learning. 26th Annual Conference on Neural Information Processing Systems 2012, (NIPS 2012), Lake Tahoe, NV, United states, December 3-6, 2012, 287-195.

17. Liu, G., Liu, Y., Guo, M. Variational Inference with Gaussian Mixture Model and Householder Flow. Neural Networks, 2019, 109, 43-55. https://doi.org/10.1016/j.neunet.2018.10.002

18. Ma, J., Zhao, J., Tian, J. Robust Point Matching via Vector Field Consensus. IEEE Transactions on Image Process, 2014, 23(4), 1706-1721. https://doi.org/10.1109/ TIP.2014.2307478

19. Minh, H. Q., Bazzani, L., Murino, V. A Unifying Framework in Vector-valued Reproducing Kernel Hilbert Spaces for Manifold Regularization and Co-Regularized Multi-view Learning. Journal of Machine Learning Research, 2016, 17.

20. Muandet, K., Fukumizu, K., Sriperumbuder, B. Kernal Mean Embedding of Distributions: A Review and Beyond. Foundations and Trends in Machine Learning, 2017, 1-141. https://doi.org/10.1561/2200000060

21. Novati, P., Gazzola, S. Multi-Parameter Arnoldi-Tikhonov Methods. Electronic Transactions on Numerical Analysis, 2013, 40, 452-475.

22. Qin, Y., Xu, H., Chen, H. Image Feature Points Matching via Improved ORB. Proceedings of the 2014 
IEEE International Conference on Progress in Informatics and Computing, (PIC 2014), Shanghai, China, May 16-18, 2014, 204-208. https://doi.org/10.1109/ PIC.2014.6972325

23. Rublee, E., Rabaud, V., Konolige, K. ORB: An Efficient Alternative to SIFT or SURF. Proceedings of the IEEE International Conference on Computer Vision, (ICCV 2011), Barcelona, Spain, November 6-13, 2011, 25642571. https://doi.org/10.1109/ICCV.2011.6126544

24. Skala, V., Smolik, M. A New Approach to Vector Field Interpolation, Classification and Robust Critical Points Detection Using Radial Basis Functions. Proceedings of 7th Computer Science On-line Conference 2018, Zlin, Czech Republic, April 25-28, 2018, 109-115. https://doi. org/10.1007/978-3-319-91192-2_12

25. Sun, L., Zhang, Y., Ma, M. Hybrid GA Variational Bayes Inference of Finite Mixture Models for Voxel Classification in Brain Images. Processing of International Conference on Smart Materials and Intelligent Systems, Chongqing, China, December17-20, 2010, 364-369. https://doi.org/10.4028/www.scientific.net/ AMR.143-144.364

26. Sun, S. Infinite mixtures of multivariate Gaussian processes. Proceedings of the 2013 International Conference on Machine Learning and Cybernetics, (ICMLC
2013), Tianjin, China, July 14-17, 2013, 1011-1016. https://doi.org/10.1109/ICMLC.2013.6890744

27. Tang, C., Wang, R., Wang, W. A New Frame Interpolation Method with Pixel-Level Motion Vector Field. 2014 IEEE Visual Communications and Image Processing Conference, Valletta, MALTA, December 07-10, 2014, 350-353. https://doi.org/10.1109/VCIP.2014.7051578

28. Wang, Y., Zheng. J., Xu, Q. An Improved RANSAC Based on the Scale Variation Homogeneity. Journal of Visual Communication and Image Representation, 2016,40(PB), 751-764. https://doi.org/10.1016/j.jvcir.2016.08.019

29. Wang, Z., Kang, J., Cheng, L. Mixed Gaussian Models for Modeling Fluctuation Process Characteristics of Photovoltaic Outputs. Frontiers in Energy Research, 2019, 7. https://doi.org/10.3389/fenrg.2019.00076

30. Yang, S., Li, B. Outliers Elimination Based Ransac for Fundamental Matrix Estimation. 2013 International Conference on Virtual Reality and Visualization, (ICVRV 2013), Xian, China, September 14-15, 2013, 321-324. https://doi.org/10.1109/ICVRV.2013.63

31. Zhao, M., Chen, H., Song, T. Research on Image Matching Based on Improved RANSAC-SIFT Algorithm. 16th International Conference on Optical Communications and Networks, (ICOCN 2017), Wuzhen, China, August 7-10, 2017, 1-3. https://doi.org/10.1109/ICOCN.2017.8121270 